\title{
SHARP ENDPOINT ESTIMATES FOR THE $X$-RAY TRANSFORM AND THE RADON TRANSFORM IN FINITE FIELDS
}

\author{
DOOWON KOH
}

(Communicated by Michael T. Lacey)

\begin{abstract}
This note establishes sharp $L^{p}-L^{r}$ estimates for $X$-ray transforms and Radon transforms in finite fields.
\end{abstract}

\section{INTRODUCTION}

Let $\mathbb{F}_{q}^{d}, d \geq 2$, be a $d$-dimensional vector space over the finite field $\mathbb{F}_{q}$ with $q$ elements. We endow $\mathbb{F}_{q}^{d}$ with a normalized counting measure $d x$. For each $q$, we denote by $M_{q}$ a collection of certain subsets of $\mathbb{F}_{q}^{d}$. Recall that a normalized surface measure $d \sigma$ on $M_{q}$ can be defined by the relation

$$
\int \Omega(w) d \sigma(w)=\frac{1}{\left|M_{q}\right|} \sum_{w \in M_{q}} \Omega(w),
$$

where $\left|M_{q}\right|$ denotes the cardinality of $M_{q}$ and $\Omega$ is a complex-valued function on $M_{q}$. For any complex-valued function $f$ on $\left(\mathbb{F}_{q}^{d}, d x\right)$ and $w \in M_{q}$, we consider an operator $T_{M_{q}}$ defined by

$$
T_{M_{q}} f(w)=\frac{1}{|w|} \sum_{x \in w} f(x) .
$$

We are interested in determining exponents $1 \leq p, r \leq \infty$ such that the following inequality holds:

$$
\left\|T_{M_{q}} f\right\|_{L^{r}\left(M_{q}, d \sigma\right)} \lesssim\|f\|_{L^{p}\left(\mathbb{F}_{q}^{d}, d x\right)} \text { for all } f \text { on } \mathbb{F}_{q}^{d},
$$

where the operator norm of $T_{M_{q}}$ is independent of $q$, the size of the underlying finite field $\mathbb{F}_{q}$. If $M_{q}$ is $\Pi_{k}$, a collection of all $k$-planes in $\mathbb{F}_{q}^{d}$ with $1 \leq k \leq d-1$, then the operator $T_{\Pi_{k}}$ is called as the $k$-plane transform. In particular, $T_{\Pi_{1}}$ and $T_{\Pi_{(d-1)}}$ are known as the $X$-ray transform and the Radon transform respectively. In Euclidean space, the complete mapping properties of $k$-plane transforms were proved by M. Christ in [1]. Readers may refer to [9, [8, 3] for the description of $k$-plane transforms in the Euclidean setting. In 2008, Carbery, Stones, and Wright [2] initially studied the mapping properties of $k$-plane transforms in finite fields. Using combinatorial arguments, they proved the following theorem.

Received by the editors November 4, 2011.

2010 Mathematics Subject Classification. Primary 43A32; Secondary 43A15, 11T99.

Key words and phrases. $k$-plane transform, $X$-ray transform, Radon transform, finite fields. 
Theorem 1.1. Let $d \geq 2$ and let $1 \leq k \leq d-1$ be an integer. If

$$
\left\|T_{\Pi_{k}} f\right\|_{L^{r}\left(\Pi_{k}, d \sigma\right)} \leq C\|f\|_{L^{p}\left(\mathbb{F}_{q}^{d}, d x\right)}
$$

holds with $C$ independent of $\left|\mathbb{F}_{q}\right|$, then $(1 / p, 1 / r)$ lies in the convex hull $H$ of

$$
((k+1) /(d+1), 1 /(d+1)),(0,0),(1,1) \text { and }(0,1) .
$$

Conversely, if $(1 / p, 1 / r)$ lies in $H \backslash\{((k+1) /(d+1), 1 /(d+1))\}$, then (1.1) holds with $C$ independent of $\left|\mathbb{F}_{q}\right|$. Finally, if $(1 / p, 1 / r)=((k+1) /(d+1), 1 /(d+1))$, then the restricted type inequality

$$
\left\|T_{\Pi_{k}} f\right\|_{L^{d+1}\left(\Pi_{k}, d \sigma\right)} \leq C\|f\|_{L^{\frac{d+1}{k+1}, 1}\left(\mathbb{F}_{q}^{d}, d x\right)}
$$

holds with $C$ independent of $\left|\mathbb{F}_{q}\right|$.

Notice from Theorem 1.1 that if one could show that the restricted type inequality (1.2) can be replaced by the strong type inequality, then the mapping properties of the $k$-plane transforms in finite fields would be completely established. Namely, our task would prove the following conjecture.

Conjecture 1.2. Let $d \geq 2$ and $1 \leq k \leq(d-1)$ be integers. Then we have

$$
\left\|T_{\Pi_{k}} f\right\|_{L^{d+1}\left(\Pi_{k}, d \sigma\right)} \leq C\|f\|_{L^{\frac{d+1}{k+1}\left(\mathbb{F}_{q}^{d}, d x\right)}} \text { for all } f \text { on } \mathbb{F}_{q}^{d},
$$

where $C$ is independent of $\left|\mathbb{F}_{q}\right|$.

1.1. Statement of the main results. In this paper we prove that Conjecture 1.2 is true for the $X$-ray transform and the Radon transform. More precisely, we obtain the following theorem.

Theorem 1.3. Let $d \geq 2$ be any integer. If $k=1$ or $k=d-1$, then

$$
\left\|T_{\Pi_{k}} f\right\|_{L^{d+1}\left(\Pi_{k}, d \sigma\right)} \leq C\|f\|_{L^{\frac{d+1}{k+1}\left(\mathbb{F}_{q}^{d}, d x\right)}} \text { for all } f \text { on } \mathbb{F}_{q}^{d},
$$

where $C$ is independent of $\left|\mathbb{F}_{q}\right|$.

In order to prove Theorem 1.3 for the $X$-ray transform $(k=1)$, we shall adapt both the combinatorial arguments in [2] and the skills in [6] for endpoint estimates. On the other hand, a Fourier analytic argument will be required to prove Theorem 1.3 for the Radon transform $(k=d-1)$.

Remark 1.4. After writing this paper, the author realized that our result for the $X$-ray transform is a corollary of Theorem 1.1 in the paper [4]. This was pointed out by R. Oberlin.

\section{Proof of the mapping properties of the $X$-RAY transform}

In this section, we restate and prove Theorem 1.3 in the case of the $X$-ray transform. Namely, we prove the following statement which implies the sharp boundedness of the $X$-ray transform.

Theorem 2.1. Let $d \geq 2$ be any integer. Then

$$
\left\|T_{\Pi_{1}} f\right\|_{L^{d+1}\left(\Pi_{1}, d \sigma\right)} \leq C\|f\|_{L^{\frac{d+1}{2}}\left(\mathbb{F}_{q}^{d}, d x\right)} \text { for all } f \text { on } \mathbb{F}_{q}^{d},
$$

where $C$ is independent of $\left|\mathbb{F}_{q}\right|$. 
Proof. We begin by following the argument in 6]. Without loss of generality, we may assume that $f$ is a non-negative real-valued function and

$$
\sum_{x \in \mathbb{F}_{q}^{d}} f(x)^{\frac{d+1}{2}}=1 .
$$

Thus, it is natural to assume that $\|f\|_{\infty} \leq 1$. Furthermore, we may assume that $f$ is written by a step function

$$
f(x)=\sum_{i=0}^{\infty} 2^{-i} E_{i}(x)
$$

where the sets $E_{i}$ are disjoint subsets of $\mathbb{F}_{q}^{d}$, and here, and throughout the paper, we write $E(x)$ for the characteristic function on a set $E \subset \mathbb{F}_{q}^{d}$.

From (2.1) and (2.2), we also assume that

$$
\sum_{j=0}^{\infty} 2^{-\frac{(d+1) j}{2}}\left|E_{j}\right|=1 \text { and }\left|E_{j}\right| \leq 2^{\frac{(d+1) j}{2}} \text { for all } j=0,1, \ldots .
$$

Since $d x$ is the normalized counting measure on $\mathbb{F}_{q}^{d}$, assumption (2.1) shows that we only need to prove

$$
\left\|T_{\Pi_{1}} f\right\|_{L^{d+1}\left(\Pi_{1}, d \sigma\right)}^{d+1} \lesssim q^{-2 d},
$$

where $f$ satisfies (2.2) and (2.3). Since we have assumed that $f \geq 0$, it is clear that $T_{\Pi_{1}} f$ is also a non-negative real-valued function on $\Pi_{1}$. By expanding the left-hand side of (2.4) and using the facts that $|w|=q$ for $w \in \Pi_{1}$ and $\left|\Pi_{1}\right| \sim q^{2(d-1)}$, we see that

$$
\begin{gathered}
\left\|T_{\Pi_{1}} f\right\|_{L^{d+1}\left(\Pi_{1}, d \sigma\right)}^{d+1}=\frac{1}{\left|\Pi_{1}\right|} \sum_{w \in \Pi_{1}}\left(T_{\Pi_{1}} f(w)\right)^{d+1} \\
\sim \frac{1}{q^{d+1}} \frac{1}{q^{2(d-1)}} \sum_{i_{0}=0}^{\infty} \cdots \sum_{i_{d}=0}^{\infty} 2^{-\left(i_{0}+\cdots+i_{d}\right)} \sum_{\left(x_{0}, \ldots, x_{d}\right) \in E_{i_{0}} \times \cdots \times E_{i_{d}}} \sum_{w \in \Pi_{1}} w\left(x_{0}\right) \cdots w\left(x_{d}\right) \\
\sim \frac{1}{q^{d+1}} \frac{1}{q^{2(d-1)}} \sum_{0=i_{0} \leq i_{1} \leq \cdots \leq i_{d}<\infty} 2^{-\left(i_{0}+\cdots+i_{d}\right)} \sum_{\left(x_{0}, \ldots, x_{d}\right) \in E_{i_{0}} \times \cdots \times E_{i_{d}}} w\left(x_{0}\right) \cdots w\left(x_{d}\right),
\end{gathered}
$$

where the last line follows from the symmetry of $i_{0}, \cdots, i_{d}$. We now follow the argument in [2]. Notice that we can write

$$
\sum_{\left(x_{0}, \ldots, x_{d}\right) \in E_{i_{0}} \times \cdots \times E_{i_{d}}}=\sum_{s=0}^{\infty} \sum_{\left(x_{0}, \ldots, x_{d}\right) \in \Delta\left(s, i_{0}, \ldots, i_{d}\right)},
$$

where $\Delta\left(s, i_{0}, \ldots, i_{d}\right)=\left\{\left(x_{0}, \ldots, x_{d}\right) \in E_{i_{0}} \times \cdots \times E_{i_{d}}:\left[x_{0}, \ldots, x_{d}\right]\right.$ is an $s$-plane $\}$ and $\left[x_{0}, \ldots, x_{d}\right]$ denotes the smallest affine subspace containing the elements $x_{0}, \ldots$, $x_{d}$. In addition, observe that if $s>1$ and $\left(x_{0}, \ldots, x_{d}\right) \in \Delta\left(s, i_{0}, \ldots, i_{d}\right)$, then the sum over $w \in \Pi_{1}$ vanishes. On the other hand, if $s=0,1$, then the sum over $w \in \Pi_{1}$ is the same as the number of lines containing the unique $s$-plane, that is, $\sim q^{(d-1)(1-s)}$. From these observations and (2.4), it is enough to prove that for all $E_{i}, i=0,1, \ldots$, satisfying (2.3),

$$
\sum_{i_{0}=0}^{\infty} \sum_{i_{1} \geq i_{0}}^{\infty} \ldots \sum_{i_{d} \geq i_{d-1}}^{\infty} 2^{-\left(i_{0}+i_{1}+\cdots+i_{d}\right)} \sum_{s=0}^{1}\left|\Delta\left(s, i_{0}, \ldots, i_{d}\right)\right| q^{-s(d-1)} \lesssim 1 .
$$


Namely, it suffices to prove that for every $d \geq 2$ and $s=0,1$,

$$
\mathrm{A}=\sum_{i_{0}=0}^{\infty} \sum_{i_{1} \geq i_{0}}^{\infty} \cdots \sum_{i_{d} \geq i_{d-1}}^{\infty} 2^{-\left(i_{0}+i_{1}+\cdots+i_{d}\right)}\left|\Delta\left(s, i_{0}, \ldots, i_{d}\right)\right| q^{-s(d-1)} \lesssim 1,
$$

where $\Delta\left(s, i_{0}, \ldots, i_{d}\right)$ is defined as before, and the sets $E_{i}, i=0,1, \ldots$, satisfy (2.3). Suppose that $s=0$. Since $\left|\Delta\left(0, i_{0}, \ldots, i_{d}\right)\right| \leq\left|E_{i_{0}}\right|$, it follows that

$$
\mathrm{A} \leq \sum_{i_{0}=0}^{\infty} \sum_{i_{1} \geq i_{0}}^{\infty} \cdots \sum_{i_{d} \geq i_{d-1}}^{\infty} 2^{-\left(i_{0}+i_{1}+\cdots+i_{d}\right)}\left|E_{i_{0}}\right| .
$$

Since the sum of a convergent geometric series is similar to the value of the first term, we have the desirable conclusion for $s=0$ :

$$
\mathrm{A} \lesssim \sum_{i_{0}=0}^{\infty}\left|E_{i_{0}}\right| 2^{-(d+1) i_{0}} \leq \sum_{i_{0}=0}^{\infty}\left|E_{i_{0}}\right| 2^{-\frac{(d+1) i_{0}}{2}}=1,
$$

where the last equality is obtained from (2.3).

Next, we assume that $s=1$. We must show that for all $E_{i}, i=0,1, \ldots$, satisfying (2.3), we have

$$
\sum_{i_{0}=0}^{\infty} \sum_{i_{1} \geq i_{0}}^{\infty} \ldots \sum_{i_{d} \geq i_{d-1}}^{\infty} 2^{-\left(i_{0}+i_{1}+\cdots+i_{d}\right)}\left|\Delta\left(1, i_{0}, \ldots, i_{d}\right)\right| q^{-(d-1)} \lesssim 1 .
$$

We estimate the upper bound of $\left|\Delta\left(1, i_{0}, \ldots, i_{d}\right)\right|$. Fix $x_{i_{0}} \in E_{i_{0}}$, which has $\left|E_{i_{0}}\right|$ choices. Notice that if $\left(x_{i_{0}}, \ldots, x_{i_{d}}\right) \in \Delta\left(1, i_{0}, \ldots, i_{d}\right)$, then all points $x_{i_{0}}, \ldots, x_{i_{d}}$ must lie on a line, which is determined by at least two different points. Therefore, for each $l=1,2, \ldots, d$, we can define

$$
\begin{array}{r}
L(l)=\left\{\left(x_{i_{0}}, \ldots, x_{i_{d}}\right) \in \Delta\left(1, i_{0}, \ldots, i_{d}\right):\left[x_{i_{0}}, \ldots, x_{i_{l}}\right]\right. \text { is a line, } \\
\text { and } \left.\left[x_{i_{0}}, \ldots, x_{i_{l-1}}\right] \text { is a point }\right\},
\end{array}
$$

where we recall that $\left[\alpha_{1}, \ldots, \alpha_{s}\right]$ means the smallest affine subspace containing all points $\alpha_{1}, \ldots, \alpha_{s}$ in $\mathbb{F}_{q}^{d}$. It is clear that $\Delta\left(1, i_{0}, \ldots, i_{d}\right)=\bigcup_{l=1}^{d} L(l)$, which implies that

$$
\left|\Delta\left(1, i_{0}, \ldots, i_{d}\right)\right| \leq \sum_{l=1}^{d}|L(l)| .
$$

By the definition of $L(l), l=1, \ldots, d$, it follows that for every $l=1, \ldots, d$,

$$
|L(l)| \leq\left|E_{i_{0}}\right|\left|E_{i_{l}}\right| q^{d-l} .
$$

To see this, first fix $x_{i_{0}} \in E_{i_{0}}$, which has $\left|E_{i_{0}}\right|$ choices. For each fixed $x_{i_{0}} \in E_{i_{0}}$, if $\left(x_{i_{0}}, \ldots, x_{d}\right) \in L(l)$, then all points $x_{i_{1}}, \ldots, x_{i_{l-1}}$ are automatically chosen as $x_{i_{0}}$, and there are at most $\left|E_{i_{l}}\right|$ choices for $x_{i_{l}} \in E_{i_{l}}$. Since $x_{0}$ and $x_{i_{l}}$ determine a fixed line, all points $x_{i_{l+1}}, \ldots, x_{i_{d}}$ must lie on the line. Thus, there are at most $q$ choices for each $x_{i_{l+1}}, \ldots, x_{i_{d}}$, because a line contains exactly $q$ points in $\mathbb{F}_{q}^{d}$.

From (2.6), (2.7), and (2.8), it suffices to prove that for every $l=1, \ldots, d$,

$$
\mathrm{B}=\sum_{i_{0}=0}^{\infty} \sum_{i_{1} \geq i_{0}}^{\infty} \cdots \sum_{i_{d} \geq i_{d-1}}^{\infty} 2^{-\left(i_{0}+i_{1}+\cdots+i_{d}\right)}\left|E_{i_{0}}\right|\left|E_{i_{l}}\right| q^{1-l} \lesssim 1 .
$$


Since $\left|E_{i_{l}}\right| \leq q^{d}$ and $l \geq 1$, it is easy to see that $\left|E_{i_{l}}\right|^{(l-1) / d} q^{1-l} \lesssim 1$. Therefore, it follows that

$$
\mathrm{B} \lesssim \sum_{i_{0}=0}^{\infty} \sum_{i_{1} \geq i_{0}}^{\infty} \cdots \sum_{i_{d} \geq i_{d-1}}^{\infty} 2^{-\left(i_{0}+i_{1}+\cdots+i_{d}\right)}\left|E_{i_{0}}\right|\left|E_{i_{l}}\right|^{\frac{d+1-l}{d}} .
$$

Since $\frac{d+1-l}{d}>0$, applying (2.3) gives

$$
\mathrm{B} \lesssim \sum_{i_{0}=0}^{\infty} \sum_{i_{1} \geq i_{0}}^{\infty} \cdots \sum_{i_{d} \geq i_{d-1}}^{\infty} 2^{-\left(i_{0}+i_{1}+\cdots+i_{d}\right)}\left|E_{i_{0}}\right| 2^{\frac{(d+1-l)(d+1) i_{l}}{2 d}} .
$$

Compute the inner summations by checking that each of them is a convergent geometric series. It follows that

$$
\mathrm{B} \lesssim \sum_{i_{0}=0}^{\infty}\left|E_{i_{0}}\right| 2^{\frac{\left(-d^{2}-d l-l+1\right) i_{0}}{2 d}} \leq \sum_{i_{0}=0}^{\infty}\left|E_{i_{0}}\right| 2^{-\frac{(d+1) i_{0}}{2}}=1,
$$

where the last equality follows from (2.3). Thus, we complete the proof of Theorem 2.1

Remark 2.2. It seems that arguments similar to those above work for settling Conjecture 1.2 but it may not be simple to estimate $\left|\Delta\left(s, i_{0}, \ldots, i_{d}\right)\right|$.

\section{Proof of mapping properties of the RAdon transform}

In this section, we prove Theorem 1.3 in the case of the Radon transform. Namely, we shall prove the following.

Theorem 3.1. Let $d \geq 2$ be any integer. Then,

$$
\left\|T_{\Pi_{d-1}} f\right\|_{L^{d+1}\left(\Pi_{d-1}, d \sigma\right)} \leq C\|f\|_{L^{\frac{d+1}{d}}\left(\mathbb{F}_{q}^{d}, d x\right)} \text { for all } f \text { on } \mathbb{F}_{q}^{d},
$$

where $C$ is independent of $\left|\mathbb{F}_{q}\right|$.

Proof. First, notice that if the dimension $d$ is two, then the statement of Theorem 3.1 follows immediately from Theorem 2.1. We therefore assume that $d \geq 3$. As before, we may assume that $f$ is a non-negative real function and

$$
\sum_{x \in \mathbb{F}_{q}^{d}}[f(x)]^{(d+1) / d}=1 .
$$

Moreover, we may assume that the function $f$ is a step function,

$$
f(x)=\sum_{i=0}^{\infty} 2^{-i} E_{i}(x),
$$

where the sets $E_{i}$ are disjoint subsets of $\mathbb{F}_{q}^{d}$. Notice that (3.1) and (3.2) imply that

$$
\sum_{j=0}^{\infty} 2^{-\frac{(d+1) j}{d}}\left|E_{j}\right|=1 \quad \text { and }\left|E_{j}\right| \leq 2^{\frac{(d+1) j}{d}} \text { for all } j=0,1, \ldots
$$

We write $\Pi_{d-1}=H \cup \Theta$, where $H$ and $\Theta$ are defined by

$$
H:=\left\{w \in \Pi_{d-1}:(0, \ldots, 0) \notin w\right\}
$$

and

$$
\Theta:=\left\{w \in \Pi_{d-1}:(0, \ldots, 0) \in w\right\}
$$


It is clear that $H$ and $\Theta$ are disjoint. Notice that we can identify $H$ with $\mathbb{F}_{q}^{d} \backslash$ $\{(0, \ldots, 0)\}$ in the sense that if $w \in H$, then there exists a unique $w^{\prime} \in \mathbb{F}_{q}^{d} \backslash$ $\{(0, \ldots, 0)\}$ such that

$$
w=\left\{x \in \mathbb{F}_{q}^{d}: w^{\prime} \cdot x=1\right\} .
$$

Thus, if $w \in H$, then we may assume that

$$
T_{\Pi_{d-1}} f(w)=\frac{1}{|w|} \sum_{x \in \mathbb{F}_{q}^{d}: w^{\prime} \cdot x=1} f(x)=\frac{1}{q^{d-1}} \sum_{x \in \mathbb{F}_{q}^{d}: w^{\prime} \cdot x=1} f(x) .
$$

On the other hand, for a fixed $w \in \Theta$, there is a unique line passing through the origin, say $L_{w}$, such that

$$
w=\left\{x \in \mathbb{F}_{q}^{d}: w^{\prime} \cdot x=0 \text { for all } w^{\prime} \in L_{w} \backslash\{(0, \ldots, 0)\}\right\} .
$$

By selecting one specific $w^{\prime} \in L_{w} \backslash\{(0, \ldots, 0)\}$ we can identify $w \in \Theta$ with the specific point $w^{\prime} \in L_{w} \backslash\{(0, \ldots, 0)\}$. Throughout this paper, we denote by $S$ the collection of the specific points, each of which is chosen from $L_{w} \backslash\{(0, \ldots, 0)\}$ for every $w \in \Theta 1$ Thus, we also assume that if $w \in \Theta$, then

$$
T_{\Pi_{d-1}} f(w)=\frac{1}{q^{d-1}} \sum_{x \in \mathbb{F}_{q}^{d}: w^{\prime} \cdot x=0} f(x),
$$

where $w^{\prime} \in S$. Since $\Pi_{d-1}=H \cup \Theta$ and $H \cap \Theta=\emptyset$, the Radon transform $T_{\Pi_{d-1}}$ can be viewed as

$$
T_{\Pi_{d-1}} f(w)=T_{0} f(w)+T_{1} f(w) \quad \text { for } w \in \Pi_{d-1},
$$

where the operators $T_{0}$ and $T_{1}$ are defined as

$$
T_{0} f(w)=\frac{\Theta(w)}{q^{d-1}} \sum_{x \in \mathbb{F}_{q}^{d}: w^{\prime} \cdot x=0} f(x)
$$

and

$$
T_{1} f(w)=\frac{H(w)}{q^{d-1}} \sum_{x \in \mathbb{F}_{q}^{d}: w^{\prime} \cdot x=1} f(x) .
$$

In order to prove Theorem 3.1] it therefore suffices to show that the following two inequalities hold:

$$
\left\|T_{0} f\right\|_{L^{d+1}\left(\Pi_{d-1}, d \sigma\right)} \lesssim\|f\|_{L^{\frac{d+1}{d}}\left(\mathbb{F}_{q}^{d}, d x\right)}
$$

and

$$
\left\|T_{1} f\right\|_{L^{d+1}\left(\Pi_{d-1}, d \sigma\right)} \lesssim\|f\|_{L}{ }_{\frac{d+1}{d}\left(\mathbb{F}_{q}^{d}, d x\right)},
$$

where the functions $f$ satisfy (3.1), (3.2), and (3.3).

\footnotetext{
${ }^{1}$ In the Euclidean setting, one can consider the set $S$ as a half part of the unit sphere. However, it is not true in general in the finite field setting. For example, if the dimension $d$ is four and $-1 \in \mathbb{F}_{q}$ is a square number, then the line $l=\left\{t(i, 1, i, 1): t \in \mathbb{F}_{q}\right\}$ does not intersect the set $\left\{x \in \mathbb{F}_{q}^{4}: x_{1}^{2}+\cdots+x_{4}^{2}=1\right\}$.
} 
3.1. Proof of inequality (3.4). Let us denote by $\chi$ the canonical additive character of $\mathbb{F}_{q}$ (see [7] or [5]). Recall that the orthogonality relation of $\chi$ holds:

$$
\sum_{s \in \mathbb{F}_{q}} \chi(a s)= \begin{cases}0 & \text { if } a \in \mathbb{F}_{q}^{*}=\mathbb{F}_{q} \backslash\{0\} \\ q & \text { if } a=0\end{cases}
$$

Using the orthogonality relation of $\chi$, we have

$$
\begin{gathered}
T_{0} f(w)=\frac{\Theta(w)}{q^{d-1}} \sum_{x \in \mathbb{F}_{q}^{d}}\left[\frac{1}{q} \sum_{s \in \mathbb{F}_{q}} \chi\left(s\left(w^{\prime} \cdot x\right)\right)\right] f(x) \\
=\frac{\Theta(w)}{q^{d}} \sum_{x \in \mathbb{F}_{q}^{d}} \sum_{s=0} \chi\left(s\left(w^{\prime} \cdot x\right)\right) f(x)+\frac{\Theta(w)}{q^{d}} \sum_{x \in \mathbb{F}_{q}^{d}} \sum_{s \in \mathbb{F}_{q}^{*}} \chi\left(s\left(w^{\prime} \cdot x\right)\right) f(x) \\
=\frac{\Theta(w)}{q^{d}} \sum_{x \in \mathbb{F}_{q}^{d}} f(x)+\frac{\Theta(w)}{q^{d}} \sum_{x \in \mathbb{F}_{q}^{d}} \sum_{s \in \mathbb{F}_{q}^{*}} \chi\left(s\left(w^{\prime} \cdot x\right)\right) f(x) \\
:=T_{0}^{\star} f(w)+T_{0}^{\star \star} f(w) .
\end{gathered}
$$

Since $\left|T_{0}^{\star} f(w)\right| \leq\|f\|_{L^{1}\left(\mathbb{F}_{q}^{d}, d x\right)}$ for all $w \in \Pi_{d-1}$, we see that

$$
\left\|T_{0}^{\star} f\right\|_{L^{d+1}\left(\Pi_{d-1}, d \sigma\right)} \leq\|f\|_{L^{1}\left(\mathbb{F}_{q}^{d}, d x\right)} \leq\|f\|_{L^{\frac{d+1}{d}}}{ }_{\left(\mathbb{F}_{q}^{d}, d x\right)},
$$

where we used the fact that $d x$ and $d \sigma$ are the normalized counting measure on $\mathbb{F}_{q}^{d}$ and the normalized surface measure on $\Pi_{d-1}$, respectively. To prove inequality (3.4), it remains to prove that for all functions $f$ satisfying (3.1), (3.2), and (3.3),

$$
\left\|T_{0}^{\star \star} f\right\|_{L^{d+1}\left(\Pi_{d-1}, d \sigma\right)} \lesssim\|f\|_{L^{\frac{d+1}{d}}\left(\mathbb{F}_{q}^{d}, d x\right)}=q^{-\frac{d^{2}}{d+1}},
$$

where the last equality follows from (3.1). We need the following lemma.

Lemma 3.2. Let $d \geq 3$. Then, for every subset $E \subset \mathbb{F}_{q}^{d}$, we have

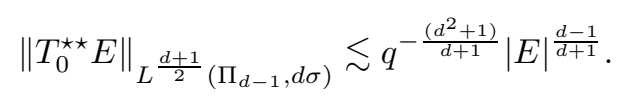

Proof. Since $d \geq 3$, we see that the statement of Lemma 3.2 follows immediately by interpolating the following two estimates: for all indicator functions $E(x)$ on $\mathbb{F}_{q}^{d}$,

$$
\left\|T_{0}^{\star \star} E\right\|_{L^{\infty}\left(\Pi_{d-1}, d \sigma\right)} \lesssim q^{-d+1}|E|
$$

and

$$
\left\|T_{0}^{\star \star} E\right\|_{L^{2}\left(\Pi_{d-1}, d \sigma\right)} \lesssim q^{-d+\frac{1}{2}}|E|^{\frac{1}{2}}
$$

To obtain (3.7), notice that

$$
\begin{gathered}
\left\|T_{0}^{\star \star} E\right\|_{L^{\infty}\left(\Pi_{d-1}, d \sigma\right)}=\left\|T_{0} E-T_{0}^{\star} E\right\|_{L^{\infty}\left(\Pi_{d-1}, d \sigma\right)} \\
\leq\left\|T_{0} E\right\|_{L^{\infty}\left(\Pi_{d-1}, d \sigma\right)}+\left\|T_{0}^{\star} E\right\|_{L^{\infty}\left(\Pi_{d-1}, d \sigma\right)} \leq q^{-d+1}|E|+q^{-d}|E| \sim q^{-d+1}|E| .
\end{gathered}
$$


It remains to prove that (3.8) holds. It follows that for every set $E \subset \mathbb{F}_{q}^{d}$,

$$
\begin{gathered}
\left\|T_{0}^{\star \star} E\right\|_{L^{2}\left(\Pi_{d-1}, d \sigma\right)}^{2}=\frac{1}{\left|\Pi_{d-1}\right|} \sum_{w \in \Pi_{d-1}}\left|\frac{\Theta(w)}{q^{d}} \sum_{x \in \mathbb{F}_{q}^{d}} \sum_{s \in \mathbb{F}_{q}^{*}} \chi\left(s\left(w^{\prime} \cdot x\right)\right) E(x)\right|^{2} \\
=\frac{1}{\left|\Pi_{d-1}\right|} \sum_{w^{\prime} \in S}\left|\frac{1}{q^{d}} \sum_{x \in E} \sum_{s \in \mathbb{F}_{q}^{*}} \chi\left(s\left(w^{\prime} \cdot x\right)\right)\right|^{2}:=\frac{1}{\left|\Pi_{d-1}\right|} \sum_{w^{\prime} \in S} \Gamma\left(w^{\prime}\right),
\end{gathered}
$$

where we used the fact that $S$ can be identified with $\Theta$. Using a change of variables, we see that for each $w^{\prime} \in S, \Gamma\left(w^{\prime}\right)=\Gamma\left(t w^{\prime}\right)$ for all $t \in \mathbb{F}_{q}^{*}$. By the definition of $S$, it therefore follows that

$$
\left\|T_{0}^{\star \star} E\right\|_{L^{2}\left(\Pi_{d-1}, d \sigma\right)}^{2} \leq \frac{1}{\left|\Pi_{d-1}\right|} \frac{1}{q-1} \sum_{w^{\prime} \in \mathbb{F}_{q}^{d}}\left|\frac{1}{q^{d}} \sum_{x \in E} \sum_{s \in \mathbb{F}_{q}^{*}} \chi\left(s\left(w^{\prime} \cdot x\right)\right)\right|^{2} .
$$

Since $\left|\Pi_{d-1}\right| \sim q^{d}$, if we expand the square term and apply the orthogonality relation of $\chi$ to the sum over $w^{\prime} \in \mathbb{F}_{q}^{d}$, we see that

$$
\begin{gathered}
\left\|T_{0}^{\star \star} E\right\|_{L^{2}\left(\Pi_{d-1}, d \sigma\right)}^{2} \lesssim \frac{1}{q^{3 d+1}} \sum_{w^{\prime} \in \mathbb{F}_{q}^{d}} \sum_{x, x^{\prime} \in E} \sum_{s, s^{\prime} \in \mathbb{F}_{q}^{*}} \chi\left(w^{\prime} \cdot\left(s x-s^{\prime} x^{\prime}\right)\right) \\
=\frac{1}{q^{2 d+1}} \sum_{x, x^{\prime} \in E, s, s^{\prime} \in \mathbb{F}_{q}^{*}: s x=s^{\prime} x^{\prime}} 1 \leq \frac{|E|}{q^{2 d-1}} .
\end{gathered}
$$

Thus, the proof of Lemma 3.2 is complete.

We now prove (3.6). Since we have assumed that $f$ is considered as a step function (3.2), it follows that

$$
\begin{aligned}
\left\|T_{0}^{\star \star} f\right\|_{L^{d+1}}^{2}\left(\Pi_{d-1}, d \sigma\right) & =\left\|\left(T_{0}^{\star \star} f\right)\left(T_{0}^{\star \star} f\right)\right\|_{L^{\frac{d+1}{2}}}\left(\Pi_{d-1}, d \sigma\right) \\
& \leq \sum_{i=0}^{\infty} \sum_{j=0}^{\infty} 2^{-i-j}\left\|\left(T_{0}^{\star \star} E_{i}\right)\left(T_{0}^{\star \star} E_{j}\right)\right\|_{L^{\frac{d+1}{2}}}\left(\Pi_{d-1}, d \sigma\right) \\
& \sim \sum_{i=0}^{\infty} \sum_{j \geq i}^{\infty} 2^{-i-j}\left\|\left(T_{0}^{\star \star} E_{i}\right)\left(T_{0}^{\star \star} E_{j}\right)\right\|_{L^{\frac{d+1}{2}}}\left(\Pi_{d-1}, d \sigma\right)
\end{aligned}
$$

where the last line follows from the symmetry of $i, j$. By Hölder's inequality, inequality (3.7), and Lemma 3.2, (3.6) will follow if we prove that

$$
\sum_{i=0}^{\infty} \sum_{j \geq i}^{\infty} 2^{-i-j}\left|E_{i}\right|\left|E_{j}\right|^{\frac{d-1}{d+1}} \lesssim 1
$$

This can be justified by making use of (3.3) and computing the summation over the $j$ variable:

$$
\sum_{i=0}^{\infty} \sum_{j \geq i}^{\infty} 2^{-i-j}\left|E_{i}\right|\left|E_{j}\right|^{\frac{d-1}{d+1}} \leq \sum_{i=0}^{\infty} \sum_{j \geq i}^{\infty} 2^{-i-j}\left|E_{i}\right|\left(2^{\frac{(d+1) j}{d}}\right)^{\frac{d-1}{d+1}} \sim \sum_{i=0}^{\infty}\left|E_{i}\right| 2^{-\frac{(d+1) i}{d}}=1
$$

which completes the proof of (3.4). 
3.2. Proof of inequality (3.5). By showing that inequality (3.5) holds, we shall complete the proof of Theorem 3.1. We shall take the same steps as in the previous subsection. From the orthogonality relation of $\chi$, it follows that for all $w \in \Pi_{d-1}$, $T_{1} f(w)=\frac{H(w)}{q^{d}} \sum_{x \in \mathbb{F}_{q}^{d}} f(x)+\frac{H(w)}{q^{d}} \sum_{x \in \mathbb{F}_{q}^{d}} \sum_{s \in \mathbb{F}_{q}^{*}} \chi\left(s\left(w^{\prime} \cdot x-1\right)\right) f(x):=T_{1}^{\star}(w)+T_{1}^{\star \star}(w)$.

As before, it is easy to see that

$$
\left\|T_{1}^{\star} f\right\|_{L^{d+1}\left(\Pi_{d-1}, d \sigma\right)} \leq\|f\|_{L^{\frac{d+1}{d}}\left(\mathbb{F}_{q}^{d}, d x\right)} .
$$

Thus, it is enough to prove that for all functions $f$ satisfying (3.1), (3.2), and (3.3),

$$
\left\|T_{1}^{\star \star} f\right\|_{L^{d+1}\left(\Pi_{d-1}, d \sigma\right)} \lesssim\|f\|_{L^{\frac{d+1}{d}}{ }_{\left(\mathbb{F}_{q}^{d}, d x\right)}}=q^{-\frac{d^{2}}{d+1}},
$$

where the last equality follows from (3.1). From the same arguments as in the proof of (3.4), our task is only to obtain Lemma 3.2 for the operator $T_{1}^{\star \star}$. As in the proof of Lemma 3.2, it suffices to prove the following two equalities: for every subset $E$ of $\mathbb{F}_{q}^{d}$,

$$
\left\|T_{1}^{\star \star} E\right\|_{L^{\infty}\left(\Pi_{d-1}, d \sigma\right)} \lesssim q^{-d+1}|E|
$$

and

$$
\left\|T_{1}^{\star \star} E\right\|_{L^{2}\left(\Pi_{d-1}, d \sigma\right)} \lesssim q^{-d+\frac{1}{2}}|E|^{\frac{1}{2}} .
$$

Inequality (3.9) follows immediately from the same argument as before. To prove (3.10), we observe that

$$
\begin{gathered}
\left\|T_{1}^{\star \star} E\right\|_{L^{2}\left(\Pi_{d-1}, d \sigma\right)}^{2}=\frac{1}{\left|\Pi_{d-1}\right|} \sum_{w \in \Pi_{d-1}}\left|\frac{H(w)}{q^{d}} \sum_{x \in \mathbb{F}_{q}^{d}} \sum_{s \in \mathbb{F}_{q}^{*}} \chi\left(s\left(w^{\prime} \cdot x-1\right)\right) E(x)\right|^{2} \\
=\frac{1}{\left|\Pi_{d-1}\right|} \sum_{w \in H}\left|\frac{1}{q^{d}} \sum_{x \in E} \sum_{s \in \mathbb{F}_{q}^{*}} \chi\left(s\left(w^{\prime} \cdot x-1\right)\right)\right|^{2} .
\end{gathered}
$$

Recall that $H \subset \Pi_{d-1}$ can be identified with $\mathbb{F}_{q}^{d} \backslash\{(0, \ldots, 0)\}$. Thus, if we dominate the sum over $w \in H$ by the sum over $w^{\prime} \in \mathbb{F}_{q}^{d}$, expand the square term, and use the orthogonality relation of $\chi$ over the variable $w^{\prime} \in \mathbb{F}_{q}^{d}$, it follows that

$$
\begin{aligned}
& 0 \leq\left\|T_{1}^{\star \star} E\right\|_{L^{2}\left(\Pi_{d-1}, d \sigma\right)}^{2} \leq \frac{1}{\left|\Pi_{d-1}\right| q^{d}} \sum_{x, x^{\prime} \in E, s, s^{\prime} \in \mathbb{F}_{q}^{*}: s x=s^{\prime} x^{\prime}} \chi\left(-s+s^{\prime}\right) \\
= & \frac{(q-1)|E|}{\left|\Pi_{d-1}\right| q^{d}}+\frac{1}{\left|\Pi_{d-1}\right| q^{d}} \sum_{x, x^{\prime} \in E, s, s^{\prime} \in \mathbb{F}_{q}^{*}: s x=s^{\prime} x^{\prime}, s \neq s^{\prime}} \chi\left(-s+s^{\prime}\right)=\mathrm{I}+\mathrm{II} .
\end{aligned}
$$

Since $\left|\Pi_{d-1}\right| \sim q^{d}$, it is clear that $\mathrm{I} \sim \frac{|E|}{q^{2 d-1}}$. We claim that $\mathrm{II} \leq 0$. Indeed, if we use a change of variables by putting $s=t, \frac{s^{\prime}}{s}=u$, then we see that

$$
\mathrm{II}=\frac{1}{\left|\Pi_{d-1}\right| q^{d}} \sum_{x, x^{\prime} \in E, t, u \in \mathbb{F}_{q}^{*}: x=u x^{\prime}, u \neq 0,1} \chi(-t(1-u)) .
$$


Since $u \neq 1$, the summation over $t \in \mathbb{F}_{q}^{*}$ is exactly -1 . Thus, our claim follows from the observation

$$
\mathrm{II}=\frac{-1}{\left|\Pi_{d-1}\right| q^{d}} \sum_{x, x^{\prime} \in E, u \in \mathbb{F}_{q}^{*}: x=u x^{\prime}, u \neq 0,1} 1 \leq 0 .
$$

Therefore, we conclude that

$$
\left\|T_{1}^{\star \star} E\right\|_{L^{2}\left(\Pi_{d-1}, d \sigma\right)}^{2} \leq \mathrm{I}+\mathrm{II} \leq \mathrm{I} \sim \frac{|E|}{q^{2 d-1}},
$$

which implies that inequality (3.10) holds. We have finished proving Theorem 3.1,

\section{REFERENCES}

1. M. Christ, Estimates for the k-plane transform, Indiana Univ. Math. J. 33 (1984), 891-910. MR763948 (86k:44004)

2. A. Carbery, B. Stones, and J. Wright, Averages in vector spaces over finite fields, Math. Proc. Camb. Phil. Soc. 144 (2008), 13-27. MR2388229 (2009d:11037)

3. S.W. Drury, Generalizations of Riesz potentials and $L^{p}$ estimates for certain $k$-plane transforms, Illinois J. Math. 28 (1984), 495-512. MR.748958 (85h:44004)

4. J.S. Ellenberg, R. Oberlin, and T. Tao, The Kakeya set and maximal conjectures for algebraic varieties over finite fields, Mathematika 56 (2010), 1-25. MR2604979(2011c:14066)

5. H. Iwaniec and E. Kowalski, Analytic Number Theory, Colloquium Publications 53, Amer. Math. Soc., Providence, RI (2004). MR2061214 (2005h:11005)

6. A. Lewko and M. Lewko, Endpoint restriction estimates for the paraboloid over finite fields, Proc. Amer. Math. Soc. 140 (2012), no. 6, 2013-2028. MR.2888189

7. R. Lidl and H. Niederreiter, Finite Fields, Cambridge University Press, Cambridge (1997). MR:1429394(97i:11115)

8. D.M. Oberlin and E.M. Stein, Mapping properties of the Radon transform, Indiana Univ. Math. J. 31 (1982), 641-650. MR667786 (84a:44002)

9. D. Solmon, A note on k-plane integral transforms, J. Math. Anal. Appl. 71 (1979), 351-358. MR.548770 (80m:44010)

Department of Mathematics, Chungbuk National University, Cheongue City, Chungbuk-Do 361-763, Republic of Korea

E-mail address: koh131@chungbuk.ac.kr 